\title{
Adesão de pacientes com diabetes mellitus ao tratamento farmacológico e não farmacológico: uma revisão integrativa da literatura
}

\author{
Adhesion of patients with diabetes mellitus to pharmacological and non-pharmacological \\ treatment: an integrative literature review
}

Adhesión de pacientes con diabetes mellitus al tratamiento farmacológico y no farmacológico: revisión integrativa de la literatura

\section{Resumo}

O presente estudo teve como objetivo identificar na literatura como acontece a adesão terapêutica no tratamento de pessoas com diabetes mellitus frente as suas fragilidades e potencialidades. Este estudo caracteriza-se por uma revisão integrativa da literatura de caráter descritivo, com abordagem qualitativa. Para sistematização na busca da coleta dos estudos e amostragem da literatura, foram utilizadas as seguintes bases de dados: BDENF, LILACS, MEDLINE, SCIELO e PubMed, por meio dos seguintes descritores: Diabetes mellitus, complicações do diabetes e qualidade de vida. Foram incluídos no estudo artigos publicados na íntegra no período de 2016 a 2021. No que se refere aos resultados, observou-se uma redução significativa da glicemia sérica em relação àqueles pacientes que fizeram uso de terapias corretas, seja ela voltada ao uso racional de medicamentos hipoglicemiantes ou através da adesão a prática de atividade física e introdução de uma alimentação correta, situação essa que conseguiu reduzir significativamente o surgimento de complicações graves e quadros de hospitalizações. Bem como também, a utilização e terapias farmacológicas e o risco de interações medicamentosas advindas de um tratamento incorreto, que muitas vezes perpassa pela deficiência na orientação médica ou pela baixa instrução educacional. Dessa forma, nota-se a necessidade de adesão por parte desses portadores de diabetes, tanto por uma qualidade de vida saudável, através da introdução de atividade física e alimentação correta, como também por meio de cartilhas de orientações que serviram de recurso para prevenção de complicações relacionadas ao descontrole dos níveis glicêmicos.

Palavras-chave: Diabetes Mellitus; Complicações do Diabetes; Qualidade de vida.

\section{Abstract}

The present study aimed to identify in the literature how the adherence to the treatment of people with diabetes mellitus happens, considering its weaknesses and potential. This study, if by an integrative literature review of descriptive character, with a qualitative approach. To systematize the search for the collection of studies and sampling of the literature, the following databases were used: BDENF, LILACS, MEDLINE, SCIELO and PubMed, using the 
following descriptors: Diabetes mellitus, diabetes complications and quality of life. Articles published in full from 2016 to 2021 were included in the study. Regarding the results, there was a reduced reduction in serum glucose in relation to those patients who used the correct therapies, whether aimed at the rational use of hypoglycemic medications or through adherence to physical activity and the introduction of a correct diet, a situation that managed to reduce the appearance of serious complications and hospitalizations. As well as the use of pharmacological therapies and the risk of drug interactions arising from incorrect treatment, which often permeates the deficiency in medical guidance or lack of educational instruction. Thus, there is a need for adherence by these people with diabetes, both for a healthy quality of life, through the introduction of activity and correct nutrition, as well as through physical guidance booklets that served as a resource for the prevention of complications related to uncontrolled blood glucose levels.

Keywords: Diabetes Mellitus; Diabetes complications; Quality of life.

\section{Resumen}

El presente estudio tuvo como objetivo identificar en la literatura cómo ocurre la adherencia al tratamiento de las personas con diabetes mellitus, considerando sus debilidades y potencialidades. Este estudio, si es mediante una revisión integradora de la literatura de carácter descriptivo, con un enfoque cualitativo. Para sistematizar la búsqueda de la colección de estudios y muestreo de la literatura se utilizaron las siguientes bases de datos: BDENF, LILACS, MEDLINE, SCIELO y PubMed, utilizando los siguientes descriptores: Diabetes mellitus, complicaciones de la diabetes y calidad de vida. En el estudio se incluyeron artículos publicados íntegramente de 2016 a 2021 . En cuanto a los resultados, se observó una disminución de la reducción de la glucosa sérica en relación a aquellos pacientes que utilizaron las terapias correctas, ya sea orientadas al uso racional de medicamentos hipoglucemiantes o por adherencia a los mismos. la actividad física y la introducción de una correcta alimentación, situación que logró reducir la aparición de complicaciones graves y hospitalizaciones. Así como el uso de terapias farmacológicas y el riesgo de interacciones medicamentosas derivadas de un tratamiento incorrecto, que a menudo permea la deficiencia en la orientación médica o la falta de instrucción educativa. Así, existe una necesidad de adherencia por parte de estas personas con diabetes, tanto para una calidad de vida saludable, a través de la introducción de actividad y una correcta nutrición, como a través de folletos de orientación física que sirvan como recurso para la prevención de complicaciones relacionadas con los niveles incontrolados de glucosa en sangre.

Palabras clave: Diabetes Mellitus; Complicaciones de la diabetes; Calidad de vida.

\section{Introdução}

O Diabetes Mellitus (DM) está inserido dentro do contexto das doenças crônicas não transmissíveis (DCNT), essa patologia caracteriza-se por uma condição metabólica marcada por quadros de hiperglicemia, devido a uma falha na produção de um hormônio chamado insulina, responsável pela regulação da glicose no sangue ou em uma situação chamada de resistência à insulina. Sua classificação se dá em subtipos: DM tipo 1, tipo 2 e gestacional, dentre esses destaca-se o tipo 2 como sendo a principal causa de mortes no mundo associada ao Diabetes Mellitus (Souza \& Oliveira, 2020).

Aliado a isso, é possível afirmar que a população mundial acometida pelo DM atualmente está estimada em aproximadamente 387 milhões, como esses números vêm crescendo no decorrer dos anos a expectativa é que em 2035 ultrapassem os 470 milhões de casos. No entanto, essa problemática pode se tornar ainda maior visto que estudos mostram que mais da metade das pessoas portadoras de DM desconhecem essa sua condição, podendo assim contribuir para um aumento da morbimortalidade decorrente da ausência de um tratamento prévio (Marques et al., 2019).

Segundo a Sociedade Brasileira de Diabetes (SBD), no Brasil, atualmente existem em média aproximadamente 13 milhões de pessoas portadoras dessa doença. Logo, esse número representa 6,9 \% de toda a população do país, nesse sentido aponta-se como fatores condicionantes para o crescimento da prevalência do DM o aumento acelerado da urbanização nos últimos anos, hábitos nutricionais errados, sobrepeso, obesidade e estilo de vida sedentário (SBD, 2019).

Tudo isso corrobora para um crescimento desenfreado no que diz respeito à morbimortalidade por Diabetes mellitus no Brasil. Visto que um estudo realizado no período de 2000 a 2015, onde foram analisando dados nacionais e do estado do Ceará, foi evidenciado que ocorreram 764.418 mortes por DM no país, ao mesmo tempo em que no estado do Ceará esses números registrados foram de 28.583 óbitos. Pode-se observar que houve um crescimento nos índices de mortalidade a nível 
nacional em contrapartida de uma diminuição estadual, bem como já era esperado, devido à proporcionalidade populacional (Garces et al., 2018).

É possível afirmar que os estudos e dados epidemiológicos supracitados mostram que a incidência e prevalência dos casos de DM no Brasil e no mundo, apresentam evolução rápida e alarmante nos últimos anos. As políticas públicas de saúde voltadas para prevenção e controle dessa problemática são de fundamental importância para que haja uma rápida redução desses índices de crescimento do Diabetes Mellitus no Brasil (SBD, 2019).

A Estratégia Saúde da Família (ESF) no âmbito da atenção primária em saúde desempenha um papel primordial no controle dessas alterações e complicações metabólicas, trabalhando com foco na melhoria da qualidade de vida para essas pessoas. Através de ações desenvolvidas não apenas no acompanhamento e monitoramento como também na distribuição de medicamentos, os quais possibilitam um tratamento adequado e reduzem complicações. Assim, os cuidados de saúde ofertados pela ESF possuem o objetivo de reduzir os índices de morbimortalidades de pacientes com DM através de orientações voltadas exclusivamente para o controle da doença (Santos et al., 2020).

Mediante ao grande índice de pacientes acometidos por essa patologia, surgiu o seguinte questionamento: Portadores de Diabetes Mellitus possuem conhecimento adequado sobre as medidas preventivas e tratamentos adequados da doença?

O presente trabalho teve como objetivo identificar na literatura a adesão as terapias farmacológicas e não farmacológicas no tratamento de pacientes com diabetes mellitus, como garantia para uma melhor qualidade de vida.

\section{Metodologia}

Trata-se de uma revisão integrativa da literatura, realizada a partir das seguintes etapas: (1) Identificação do tema e definição da pergunta; (2) estabelecimento de critérios para inclusão e exclusão dos estudos; (3) definição das informações a serem extraídas dos estudos selecionados; (4) avaliação dos estudos; (5) interpretação dos resultados e (6) síntese do conhecimento (Mendes et al., 2008). Para identificação do tema e definição da pergunta, foi utilizada a estratégia PICo (PPopulação: Portadores de Diabetes Mellitus; I- Interesse: Complicações do diabetes; Cocontexto: Qualidade de vida). Tendo, assim, como pergunta norteadora: "Portadores de Diabetes Mellitus possuem conhecimento adequado sobre as medidas preventivas e tratamentos adequados da doença?"

A busca foi realizada nos meses de maio a junho de 2021, nas bases de dados: Bases de Dados em Enfermagem (BDENF), Literatura Latino-Americano e do Caribe em Ciência da Saúde (LILACS), National Library Medicine (MEDLINE), Scientific Electronic Library Online (SCIELO) e PubMed por meio da Plataforma da Coordenação de Aperfeiçoamento de Pessoal de Nível Superior (CAPES). Foi realizada uma consulta nos Descritores em Ciências da Saúde (DECS), em que se utilizou os seguintes descritores: Portadores de Diabetes Mellitus, Complicações do diabetes e Qualidade de vida, associados ao operador boleano "AND" como estratégia de busca.

O cruzamento dos descritores obteve 970 publicações. Logo em seguida, iniciou-se a realização da segunda etapa da revisão, na qual foram adicionados os critérios de inclusão: artigos publicados na íntegra no período de 2016 a 2021, escritos na língua portuguesa e que atendessem aos descritores estando disponíveis gratuitamente para leitura na íntegra, alcançando 170 produções. Depois, foram inseridos os critérios de exclusão, artigos de revisão, estudos que não abordavam a temática proposta, estudos indisponíveis na integra e artigos por repetições, restando, assim, 16 artigos. Após leitura na íntegra, e análise dos objetivos e resumos, foram elegíveis 11 artigos para compor esta revisão. Além disso, foi utilizada a estratégia Preferred Reporting Items for Systematic reviews and Meta-Analyses (PRISMA) (Moher et al., 2009) - para seleção das publicações, pois os estudos se organizavam em quatro passos: identificação, seleção, elegibilidade e inclusão (Figura 1). 
Figura 1 - Fluxograma do percurso de seleção dos estudos a partir das recomendações PRISMA.

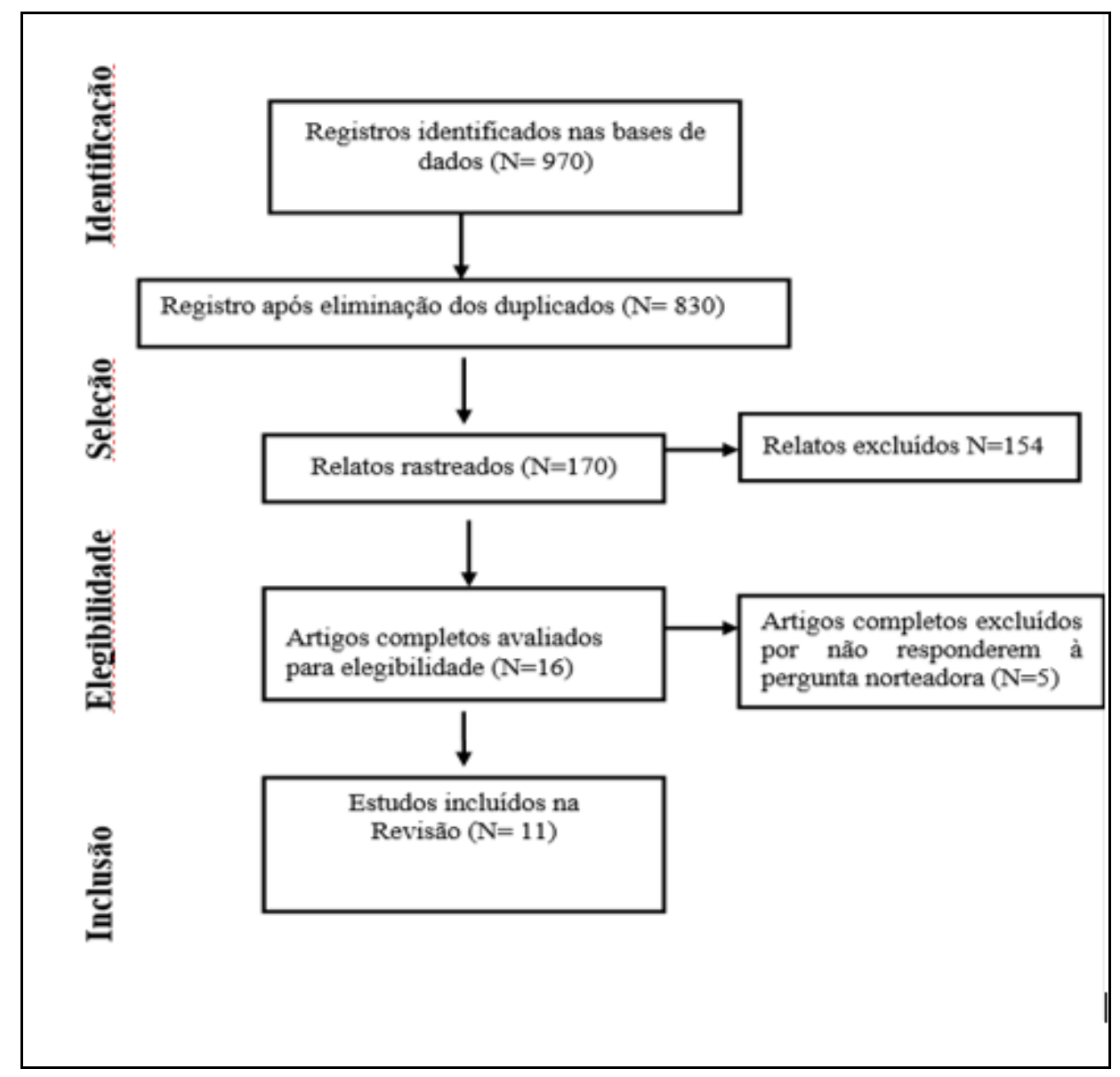

Fonte: Dados da pesquisa (2021).

$\mathrm{Na}$ terceira etapa de definição das informações a serem extraídas dos estudos selecionados, os artigos incluídos foram analisados em uma tabela do Microsoft Word® 2016, agrupados e organizados pelos seguintes itens: Identificação, título, autor, periódico, ano de publicação e resultado. Dessa forma, foi possível realizar a quarta, quinta e sexta etapas, mediante avaliação, análise e interpretação dos artigos incluídos conforme literatura científica pertinente e atualizada na área para, e dessa forma, realizar a apresentação desta revisão.

\section{Resultados e Discussão}

Os artigos científicos inseridos neste estudo, estão apresentados no Quadro 1. Logo após apresentação do quadro, estão apresentados os principais resultados obtidos a partir dos artigos selecionados por meio de categorias temáticas, as quais nortearam a produção do conhecimento a respeito da adesão de pacientes portadores de Diabetes Mellitus ao tratamento farmacológico e não farmacológico. 
Quadro 1 - Artigos selecionados para a revisão integrativa da literatura, nas bases de dados online, entre 2016-2021.

\begin{tabular}{|c|c|c|c|c|c|}
\hline $\mathbf{N}^{0}$ & Título & Periódico & Autor & Ano & Resultado \\
\hline I & $\begin{array}{l}\text { Tendência do uso e fontes } \\
\text { de obtenção de } \\
\text { antidiabéticos orais para } \\
\text { tratamento de diabetes no } \\
\text { Brasil de } 2012 \text { a 2018: } \\
\text { Análise do inquérito } \\
\text { Vigitel. }\end{array}$ & $\begin{array}{l}\text { Revista } \\
\text { Brasileira de } \\
\text { Epidemiologia }\end{array}$ & $\begin{array}{l}\text { Leitão, V. B. G. } \\
\text { et al. }\end{array}$ & 2021 & $\begin{array}{l}\text { Observa-se um aumento na prevalência do uso de } \\
\text { medicamentos orais para o tratamento do diabetes, } \\
\text { passando de } 77,4 \text { para } 85,2 \% \text {, entre } 2012 \text { e } 2018 \text {. Essas } \\
\text { informações indicam que houve diminuição nas } \\
\text { desigualdades regionais de acesso a medicamentos para } \\
\text { diabetes, um fator que pode contribuir para esse resultado } \\
\text { é a possibilidade de obtenção gratuita dos antidiabéticos } \\
\text { orais. }\end{array}$ \\
\hline II & $\begin{array}{l}\text { Adesão ao tratamento de } \\
\text { diabetes mellitus e relação } \\
\text { com a assistência na } \\
\text { atenção primária. }\end{array}$ & $\begin{array}{l}\text { Revista Mineira } \\
\text { de Enfermagem }\end{array}$ & $\begin{array}{l}\text { Santos, A. L. et } \\
\text { al. }\end{array}$ & 2020 & $\begin{array}{l}\text { Avalia que } 408 \text { pessoas participantes do estudo tinham } \\
\text { idade média de } 66,5 \text { anos. Desses } 84,1 \% \text { relataram aderir } \\
\text { ao tratamento medicamentoso, } 29,4 \% \text { realizavam } \\
\text { atividade física regularmente e apenas } 24 \% \text { tinham } \\
\text { alimentação adequada. }\end{array}$ \\
\hline III & $\begin{array}{l}\text { Aproximação dialógica às } \\
\text { necessidades de saúde em } \\
\text { usuários de insulina } \\
\text { acompanhados } \\
\text { Programa no } \\
\text { Automonitoramento } \\
\text { Glicêmico. }\end{array}$ & $\begin{array}{l}\text { Interface } \\
\text { (Botucatu) }\end{array}$ & $\begin{array}{l}\text { Dias, I. W. H. et } \\
\text { al }\end{array}$ & 2020 & $\begin{array}{l}\text { Aponta-se que o controle metabólico do DM se deu a } \\
\text { partir da consideração do índice de hemoglobina glicada } \\
\text { referente aos últimos três meses da participação do estudo } \\
\text { de campo. Na primeira roda de conversa, esse índice } \\
\text { variou entre } 7,9 \text { a } 12,5 \% \text { e média de } 8,53 \% \text {. Na segunda, } \\
\text { encontrou-se uma variação entre } 6,8 \text { a } 12,3 \% \text { e média de } \\
9,05 \% \text {. }\end{array}$ \\
\hline IV & $\begin{array}{l}\text { Avaliação da cartilha para } \\
\text { orientação da prática do } \\
\text { autocuidado em } \\
\text { Diabetes Mellitus. }\end{array}$ & $\begin{array}{ll}\text { Revista } & \text { de } \\
\text { Enfermagem } & \text { da } \\
\text { UERJ, Rio } & \text { de } \\
\text { Janeiro. } & \end{array}$ & $\begin{array}{l}\text { Torres, H. C. et } \\
\text { al }\end{array}$ & 2019 & $\begin{array}{l}\text { As figuras ilustrativas representam elementos importantes } \\
\text { nos materiais educativos, por facilitarem a compreensão e } \\
\text { motivarem o público a manusear esse material. As figuras } \\
\text { possibilitam que o cliente visualize a importância da } \\
\text { atividade física e do plano alimentar em uma estrutura } \\
\text { não linear e flexível, aumentando a possibilidade do } \\
\text { usuário em efetivar a prática do autocuidado no seu } \\
\text { cotidiano, favorecendo não apenas o interesse pelo } \\
\text { assunto como também o aprendizado. }\end{array}$ \\
\hline $\mathbf{V}$ & $\begin{array}{l}\text { Mapa de conversação } \\
\text { como estratégia de ensino } \\
\text { para o desenvolvimento do } \\
\text { autocuidado em diabetes } \\
\text { mellitus }\end{array}$ & $\begin{array}{l}\text { Enfermagem } \\
\text { Foco }\end{array}$ & $\begin{array}{l}\text { Barduchi, R. I., } \\
\text { et al. }\end{array}$ & 2019 & $\begin{array}{l}\text { Observou-se que o } \\
\text { possibilitou adesão a alimentação específica, atividade } \\
\text { física, monitorização da glicemia e cuidados com os pés } \\
\text { dos participantes, levando a inferir que esse hábito pode } \\
\text { proporcionar redução do risco de } \\
\text { complicações decorrentes da doença. }\end{array}$ \\
\hline VI & $\begin{array}{l}\text { Adesão ao tratamento } \\
\text { dietético em portadores de } \\
\text { diabetes mellitus assistidos } \\
\text { pela estratégia saúde da } \\
\text { família }\end{array}$ & Braspen $\mathrm{J}$ & $\begin{array}{l}\text { Santos, T. B. M. } \\
\text { et al }\end{array}$ & 2018 & $\begin{array}{l}\text { O estudo mostra que os portadores de DM do Tipo } 1 \text { e } 2 \\
\text { realizavam } 6 \text { refeições ao dia }(62,5 \%) \text {. E os portadores de } \\
\text { DM tipo } 1 \text { apresentavam hábitos alimentares mais } \\
\text { saudáveis, vez que mastigavam bem os alimentos } \\
(82,4 \%) \text {, não consumiam alimentos açucarados }(58,8 \%) \text {, } \\
\text { consumiam frango sem pele }(88,2 \%) \text { e não consumiam } \\
\text { carne com gordura }(88,2 \%) \text {, apresentando assim uma alta } \\
\text { taxa de adesão, que também estava relacionada a } \\
\text { participação de grupos de diabéticos, bem como consultas } \\
\text { regulares ao médico. }\end{array}$ \\
\hline VII & $\begin{array}{l}\text { Exercício físico e diabetes } \\
\text { mellitus: } \quad \text { Explorando } \\
\text { efeitos e implicações }\end{array}$ & $\begin{array}{l}\text { Acta Medica- } \\
\text { Ligas } \\
\text { acadêmicas }\end{array}$ & Paludo, L. et al. & 2018 & 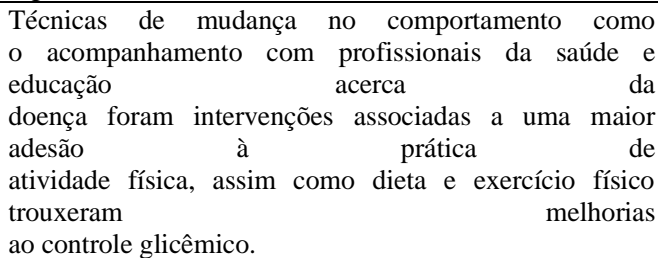 \\
\hline VIII & $\begin{array}{l}\text { Nível de atividade física } \\
\text { em pessoas com diabetes } \\
\text { mellitus tipo } 2\end{array}$ & $\begin{array}{l}\text { Revista } \\
\text { Cuidarte }\end{array}$ & $\begin{array}{l}\text { Kolchraiber, F. } \\
\text { C. et al. }\end{array}$ & 2018 & $\begin{array}{l}\text { A idade média dos usuários foi de } 59 \text { anos, } \\
\text { predominância sexo feminino e não praticantes de } \\
\text { atividade física. Valores glicêmicos obtidos pelo exame } \\
\text { da hemoglobina glicada foram, } 8,1 \% \text { em centro de } \\
\text { referência e } 9,6 \% \text { em unidade básica de saúde ( } p=0,017) \text {, } \\
\text { diferenciais de controle metabólico e distintas realidades } \\
\text { de atendimento. O nível de atividade física aliado a } \\
\text { mudanças no estilo de vida e adesão terapêutica é parte } \\
\text { fundamental para o controle do diabetes e prevenção de } \\
\text { complicações, devendo ser encorajadas pelos } \\
\text { profissionais da saúde. }\end{array}$ \\
\hline IX & $\begin{array}{l}\text { Acesso e adesão a } \\
\text { medicamentos } \quad \text { entre } \\
\text { pessoas com diabetes no } \\
\text { Brasil: Evidências da } \\
\text { PNAUM }\end{array}$ & $\begin{array}{l}\text { Revista } \\
\text { Brasileira de } \\
\text { Epidemiologia }\end{array}$ & $\begin{array}{l}\text { Meiners, } \\
\text { M.M.M.D.A. et } \\
\text { al. }\end{array}$ & 2017 & $\begin{array}{l}\text { Encontrou-se uma maior proporção de mulheres, de } \\
\text { maiores de } 60 \text { anos e da classe econômica C. A maioria } \\
\text { referiu ter duas ou mais comorbidades além do diabetes e } \\
\text { tomar cinco ou mais medicamentos. Quanto ao acesso, } \\
97,8 \% \text { dizem ter acesso aos medicamentos prescritos para } \\
\text { diabetes e } 70,7 \% \text {, que os obtêm de forma totalmente } \\
\text { gratuita. }\end{array}$ \\
\hline $\mathbf{X}$ & $\begin{array}{l}\text { Efeitos terapêuticos do } \\
\text { consumo de fibra dietética }\end{array}$ & $\begin{array}{l}\text { Experimental } \\
\text { and Therapeutic }\end{array}$ & Chen, C. et al & 2016 & $\begin{array}{l}\text { O consumo regular de fibras dietéticas solúveis levou a } \\
\text { melhorias significativas nos níveis de glicose no sangue, }\end{array}$ \\
\hline
\end{tabular}




\begin{tabular}{|l|l|l|l|l|l|}
\hline & $\begin{array}{l}\text { solúvel no diabetes } \\
\text { mellitus tipo 2. }\end{array}$ & medicine & & $\begin{array}{l}\text { resistência à insulina e perfis metabólicos, sem afetar a } \\
\text { função secretora das ilhotas de Langerhans, durante um } \\
\text { período de intervenção de curto prazo em pacientes com } \\
\text { DM2. }\end{array}$ \\
\hline XI & $\begin{array}{l}\text { Diabetes em idosos: uso de } \\
\text { medicamentos } \\
\text { e risco de interação } \\
\text { medicamentosa }\end{array}$ & $\begin{array}{l}\text { Ciência \& Saúde } \\
\text { Coletiva }\end{array}$ & $\begin{array}{l}\text { Prado, M. A. M. M. } \\
\text { B. et al }\end{array}$ & 2016 & $\begin{array}{l}\text { Aponta-se que a utilização dos medicamentos de forma } \\
\text { errada pode induzir interações medicamentosas passíveis } \\
\text { de complicações graves, podendo ir desde uma diminuição } \\
\text { ou aumento do efeito terapêutico desses fármacos, como } \\
\text { também gerar resultados tóxicos e até mesmo irreversíveis } \\
\text { ao organismo desses pacientes. }\end{array}$ \\
\hline
\end{tabular}

Fonte: Dados da pesquisa (2021)

Como resultados da análise dos resultados surgiram duas categorias temáticas: "Utilização de terapias farmacológicas e promoção do autocuidado" e "Uso de terapias não farmacológicas e qualidade de vida".

\section{Categoria 1: Utilização de terapias farmacológicas e promoção do autocuidado}

No que se refere ao tratamento medicamentoso paralelo a adoção de prática do autocuidado em pacientes com diabetes mellitus, é possível afirmar que os estudos obtidos nessa revisão demonstram as diferentes características e possíveis alterações inerentes a adesão medicamentosa. Esses fatores atípicos estão diretamente relacionados à forma de utilização desses fármacos, ao grau de instrução educacional e as particularidades presentes no cuidado individual de cada um.

Os estudos de número I e II demonstram que a utilização de medicamentos orais para o tratamento do diabetes, vem crescendo nos últimos anos. Proporcionando um crescimento positivo em relação aos anos anteriores, passando de 77,4 para $85,2 \%$ entre os anos de 2012 a 2018. Isso pode ser compreendido pelo fato da obtenção de forma gratuita desses fármacos, o que impulsiona uma redução significativa no que diz respeito às desigualdades regionais de saúde.

Em estudo realizado por Nóbrega e colaboradores (2019) a utilização de antidiabéticos orais, proporciona diminuição na incidência de complicações a nível metabólico, sejam elas agudas ou crônicas, uma vez que a terapêutica de cunho farmacológico contribui para que o paciente adquira estabilidade da glicemia sérica. Levando em consideração que o quadro de hiperglicemia provoca aterosclerose nos vasos sanguíneos, condição essa, que leva ao surgimento de doenças cardíacas como a principal causa de mortes entre os portadores de diabetes mellitus.

Essas complicações por muitas vezes podem ser evitadas, primordialmente através da utilização correta desses fármacos, situação essa que está atrelada diretamente a prática do autocuidado. Nesse contexto, estudos têm demonstrado que a orientação médica em consonância com o acompanhamento regular desses pacientes e práticas de cuidados pessoais, mostraram-se como sendo ferramentas importantes no combate ao uso irracional de medicamentos e possíveis interações medicamentosas (Santos \& Senger, 2019).

No estudo de número XI é possível compreender que a utilização de medicamentos para o tratamento do diabetes mellitus de forma errada, pode induzir o paciente a apresentar interações medicamentosas, muitas vezes passíveis de complicações graves. Podendo ir desde uma diminuição ou aumento do efeito terapêutico desses fármacos, como também podem gerar resultados tóxicos e até mesmo irreversíveis ao organismo desses pacientes.

Nessa perspectiva, durante o tratamento farmacológico a utilização de dois ou mais medicamentos podem ser indicados com o objetivo de potencializar o efeito farmacológico, aumentando ainda mais a eficácia de seu tratamento. No entanto, esse recurso metodológico pode provocar uma severa toxicidade no organismo desses pacientes, bem como, a ausência na eficácia dos fármacos. Assim, entra em evidência a polifarmácia, sendo uma situação pela qual o indivíduo passa a fazer uso de cinco ou mais medicamentos em decorrência da multiplicidade de agravos à saúde (Santos \& Senger, 2019).

Essa associação ao uso de vários fármacos aumenta o potencial de erros perante as prescrições médicas, intensificando o surgimento das interações medicamentosas, visto que, os riscos de efeitos adversos tornam-se significativamente mais 
elevados em relação a usuários que não fazem uso de combinações farmacológicas durante o seu tratamento, aumentando a probabilidade da baixa adesão ao tratamento e maximizando as chances de morbimortalidade (Zanatta et al., 2020).

No que diz respeito à prática do autocuidado, o estudo de número IV evidencia a importância da utilização de cartilhas de orientações como elementos essenciais para o tratamento do diabetes mellitus. Visto que essa metodologia de ensino proporciona ao paciente maior praticidade na compreensão acerca do seu tratamento, ao passo que permite a ele vislumbrar a importância não apenas do tratamento medicamentoso, como também da atividade física e do plano alimentar.

Dessa forma, a obtenção de conhecimento a respeito da doença permite ao indivíduo aderir aos diferentes recursos terapêuticos existentes para o seu tratamento, potencializando a prática do autocuidado e minimizando as chances de complicações graves. Tendo em vista que os cuidados pessoais associados a uma alimentação saudável possibilitam maior estabilidade dos níveis glicêmicos bem como uma diminuição significativa dos riscos cardiovasculares (Borba et al., 2018).

\section{Categoria 2: Uso de terapias não farmacológicas e qualidade de vida}

É notável que, entre os estudos reunidos nesta revisão e em associação a educação em saúde, algumas medidas acabam se tornando um fator modificador do processo saúde-doença. Nesse sentindo, ao analisar as produções que trabalharam a adesão ao tratamento não medicamentoso do diabetes, foi possível constatar que o público-alvo dessas pesquisas apresentaram diversas formas para melhoria da qualidade de vida.

Os estudos de números VI e VII mencionam tanto a adesão ao tratamento dietético como a prática do exercício físico como alternativas não medicamentosas que corroboram com a melhoria da qualidade de vida para esses pacientes. Percebeu-se nestes estudos que os pacientes apresentavam uma alimentação saudável, com realização de 5 a 6 refeições diárias, mastigavam bem os alimentos, não consumiam alimentos açucarados e gordurosos, além disso participavam de grupos de apoio, orientação e acompanhamento regular com equipe multiprofissional, o que impulsionava ainda mais essa adesão.

A educação alimentar é essencial em relação ao processo de transformação, recuperação e promoção de hábitos alimentares saudáveis, visto que é capaz de garantir aprendizagem fundamental para tomada de decisão, gerando condutas, hábitos e práticas alimentares saudáveis e diversificadas (Bueno et al., 2011).

Segundo Estrela et al. (2017), a aceitação às orientações é primordial para a modificação do estilo de vida, visto que essas alterações no hábito alimentar não se limitam apenas as alterações do consumo de alimentos e a prática de atividade física, contudo interferem no aglomerado de significados ligados ao comer, ao corpo e ao viver.

Observou-se também que, após a introdução do hábito da prática de atividade física regularmente, por parte dos portadores de diabetes mellitus, houve mudanças significativas no estilo de vida dessas pessoas. Caracterizando essa adesão terapêutica como uma ferramenta importantíssima tanto para o controle dos níveis glicêmicos como também na prevenção de diversas complicações.

Nesse sentido, estudos apontam que a prática de atividade física está diretamente relacionada com a melhoria da capacidade funcional do indivíduo, bem como também ao fortalecendo do seu sistema imunológico. Demonstrando resultados positivos na condução do tratamento não farmacológico, essa metodologia ativa propicia redução significativa no que diz respeito à morbimortalidade por doenças crônicas não transmissíveis ao longo dos anos, como é o caso do diabetes mellitus (Santos \& Matsudo, 2018).

Sendo assim, a procura em manter um estilo de vida saudável se apresentou como recurso primordial na estabilidade do controle glicêmico frente a essa patologia. Visto que, essa doença por muitas vezes desencadeia no seu portador complicações a nível metabólico, que se não tratadas podem levar ao surgimento de sequelas irreversíveis. Dessa forma, a introdução de atividade física diária na vida desses pacientes, contribui para o controle da glicemia sérica, ao passo que reduz significativamente as chances de complicações graves (Lima \& Cardoso, 2019). 
$\mathrm{O}$ estudo de número $\mathrm{X}$ relata o efeito terapêutico após o consumo de fibra alimentar solúvel, onde após estudo monitorado com mais de 100 portadores de diabetes, observou-se após a intervenção que os níveis de glicose no sangue, insulina em jejum, lipoproteína, índice de resistência à insulina, melhoraram significativamente, bem como os níveis do peptídeo C, triglicerídeos e albumina sérica.

De acordo com a Associação Americana de Diabetes (ADA), pacientes que possuem o hábito de conduzir suas refeições diárias mantendo um controle da quantidade e dos tipos de carboidratos consumidos, conseguiram proporcionar maior adesão ao controle da glicemia pós-prandial e, consequentemente, para evitar o desenvolvimento de complicações advindas do diabetes.

Nesse sentido, a busca pela adoção de hábitos alimentares por pacientes com diabetes mellitus tem sido de fundamental importância, em vista de sua influência sobre o controle glicêmico e a prevenção de doenças cardiovasculares. Sua adoção pode resultar em redução de 1 a $2 \%$ nos níveis de hemoglobina glicada (Brasil, 2013).

Dessa forma, foi possível correlacionar tanto à prática de atividade física regular como a adoção de um estilo de vida saudável através da mudança nos hábitos alimentares, como sendo ações necessárias para a promoção da saúde e qualidade de vida dessas pessoas. Tendo em vista que, pacientes portadores do diabetes mellitus que não praticam atividade física regularmente ou não fazem uso de uma dieta equilibrada, apresentam menor adesão ao seu tratamento e maior risco no desenvolvimento de alterações graves a nível glicêmico (Marques et al., 2019).

\section{Considerações Finais}

Portanto, pode-se concluir que para manter a constância na estabilidade dos níveis glicêmicos e evitar complicações advindas do diabetes é imprescindivel a utilização de uma terapia correta, onde foi observado que a utilização de medicamentos orais como terapia farmacológica, contribui de forma positiva para o tratamento do diabetes, promovendo controle glicêmico e diminuindo as chances de internações hospitalares e casos de óbito.

Vale ressaltar que o uso incorreto ou associação indevida de medicamentos para o tratamento do diabetes, pode induzir o paciente a apresentar complicações graves, como é o caso das interações medicamentosas. Sendo de fundamental importância a existência de orientações, sejam elas médicas ou farmacêuticas. Como também, a obtenção de conhecimento a respeito da utilização desses fármacos, o qual muita das vezes é fornecida através de cartilhas de orientações.

No que diz respeito à utilização de terapias não farmacológicas, foi possível concluir que a introdução de uma alimentação saudável e práticas de atividade física estão associadas à melhoria da qualidade de vida, promovendo redução de quadros hiperglicêmicos e prevenindo doenças cardiovasculares.

Percebe-se a relevância do estudo e a necessidade de novas pesquisas que possam contribuir para disseminação e reflexão do tema, aumentando assim à adesão de portadores de diabetes ao uso racional de medicamentos e a introdução de hábitos saudáveis que venham a proporcionar melhor qualidade de vida e sobrevida para essas pessoas.

Por fim, destaca-se, a necessidade da criação de projetos direcionados para a educação em saúde, onde haja mobilização de mídias sociais, e assim ajudaria a alcançar um maior número de pessoas, disseminando o conhecimento, e dessa forma estimularia os portadores de diabetes acerca das suas rotinas, hábitos, alimentação e autocuidado, para melhorar a qualidade de vida.

\section{Referências}

Borba, A. K. D. O. T., Marques, A. P. D. O., Ramos, V. P., Leal, M. C. C., Arruda, I. K. G. D., \& Ramos, R. S. P. D. S. (2018). Fatores associados à adesão terapêutica em idosos diabéticos assistidos na atenção primária de saúde. Ciência \& Saúde Coletiva, 23, 953-961. 
Brasil, Ministério da Saúde. Secretaria de atenção à saúde. Departamento de Atenção Básica. Caderno de Atenção Básica: Diabetes Mellitus. Brasília, DF: Ministério da Saúde, 2013.

Bueno, J. M., Leal, F. S., Saquy, L. P. L., Santos, C. B. D., \& Ribeiro, R. P. P. (2011). Educação alimentar na obesidade: adesão e resultados antropométricos. Revista de Nutrição, 24, 575-584.

Chen, C., Zeng, Y., Xu, J., Zheng, H., Liu, J., Fan, R., \& Wang, J. (2016). Efeitos terapêuticos do consumo de fibra alimentar solúvel no diabetes mellitus tipo 2. Medicina experimental e terapêutica, 12 (2), 1232-1242.

Dias, I. W. H., \& Junqueira, V. (2020). Aproximação dialógica às necessidades de saúde em usuários de insulina acompanhados no Programa de Automonitoramento Glicêmico. Interface-Comunicação, Saúde, Educação, 24.

Estrela, K. C. A., Alves, A. C. D. C., Gomes, T. T., \& Isosaki, M. (2017). Adesão às orientações nutricionais: uma revisão de literatura. Demetra: alimentação, nutrição \& saúde, 12(1), 249-274.

Garces, T. S., Moreira, T. M. M., Sousa, G. J. B., Pereira, M. L. D., Cestari, V. R. F., Almeida, Í. L. S. D., \& Marques, A. D. B. (2018). Tendência de mortalidade por diabetes mellitus. Rev. enferm. UFPE on line, 3231-3238.

Kolchraiber, F. C., de Souza Rocha, J., César, D. J., de Oliveira Monteiro, O., Frederico, G. A., \& Gamba, M. A. (2018). Nível de atividade física em pessoas com diabetes mellitus tipo 2. Revista Cuidarte, 9(2), 2105-2116.

Leitão, V. B. G., Francisco, P. M. S. B., Malta, D. C., \& Costa, K. S. (2021). Tendência do uso e fontes de obtenção de antidiabéticos orais para tratamento de diabetes no Brasil de 2012 a 2018: análise do inquérito Vigitel. Revista Brasileira de Epidemiologia, 24.

Lima, A. P., \& Cardoso, F. B. (2019). Atividade física de lazer em idosos com diabetes tipo 2: estudo de base populacional. LICERE-Revista do Programa de Pós-graduação Interdisciplinar em Estudos do Lazer, 22(2), 1-17.

Marques, M. B., Coutinho, J. F. V., Martins, M. C., Lopes, M. V. D. O., Maia, J. C., \& Silva, M. J. D. (2019). Intervenção educativa para a promoção do autocuidado de idosos com diabetes mellitus. Revista da Escola de Enfermagem da USP, 53.

Meiners, M. M. M. D. A., Tavares, N. U. L., Guimarães, L. S. P., Bertoldi, A. D., Pizzol, T. D. S. D., Luiza, V. L., \& Merchan-Hamann, E. (2017). Acesso e adesão a medicamentos entre pessoas com diabetes no Brasil: evidências da PNAUM. Revista Brasileira de Epidemiologia, 20, 445-459.

Mendes, K. D. S., Silveira, R. C. D. C. P., \& Galvão, C. M. (2008). Revisão integrativa: método de pesquisa para a incorporação de evidências na saúde e na enfermagem. Texto \& contexto-enfermagem, 17, 758-764.

Moher, D., Liberati, A., Tetzlaff, J., Altman, DG, \& Prisma Group. (2009). Itens de relatório preferidos para revisões sistemáticas e meta-análises: a declaração PRISMA. Medicamento PLoS , 6 (7), e1000097.

Nóbrega, L. M. B., Oliveira, P. S. D., Santos, C. L. J. D., Ferreira, T. M. C., Ferreira, J. D. L., Nascimento, W. S. D., \& Costa, M. M. L. (2019). Características e qualidade de vida de pessoas com diabetes. Rev. enferm. UFPE on line, 1243-1252.

Ohl, R. I. B., Chavaglia, S. R. R., Gomes, J. L. G. C., de Oliveira Freitas, M. A., \& Gamba, M. A. (2019). Mapa de conversação como estratégia de ensino para o desenvolvimento do autocuidado em diabetes mellitus. Enfermagem em Foco, 10(2).

Paludo, L., Severo, P. R. F., Borges, V. S., Wender, I. O., Silveira, G. D. V., Pimentel, E. E., \& Schreiner, L. R. (2018). Exercício físico e diabetes mellitus: explorando efeitos e implicações. Acta méd., 109-119.

Prado, M. A. M. B. D., Francisco, P. M. S. B., \& Barros, M. B. D. A. (2016). Diabetes em idosos: uso de medicamentos e risco de interação medicamentosa. Ciência \& Saúde Coletiva, 21, 3447-3458.

Santos, A. L., Marcon, S. S., Teston, E. F., Back, I. R., Lino, I. G. T., Batista, V. C., \& Haddad, M. D. C. F. L. (2020). Adesão ao tratamento de diabetes Mellitus e relação com a assistência na atenção primária. Revista Mineira de Enfermagem, 24, 1-10.

Santos, M., \& Matsudo, V. K. R. (2018). Atividade física e uso de medicamentos. Diagn. tratamento, 152-159.

Santos, T. B. M. D., Freitas, B. D. J., \& de Almendra, S. (2018). Adesão ao tratamento dietético em portadores de diabetes mellitus assistidos pela estratégia saúde da família. Braspen J, 76-85.

Santos, A. R., \& Senger, F. R. (2019). Avaliação da terapia medicamentosa de pacientes idosos portadores de hipertensão arterial sistêmica e diabetes mellitus tipo 2 atendidos em uma unidade básica de saúde no município de Xanxerê - SC. Rev. Epidemiol. Controle Infecç, 9, 2, 155-160.

Sociedade Brasileira De Diabetes. Diretrizes da Sociedade Brasileira de Diabetes 2019-2020.: https://www.diabetes.org.br/profissionais/images/DIRETRIZES-COMPLETA-2019-2020.pdf

Souza, C. L., \& Oliveira, M. V. (2020). Fatores associados ao descontrole glicêmico de diabetes mellitus em pacientes atendidos no Sistema Único de Saúde no Sudoeste da Bahia. Cadernos Saúde Coletiva, 28, 153-164.

Torres, H. D. C., \& Paula, D. V. D. (2019). Avaliação da cartilha para orientação da prática do autocuidado em Diabetes Mellitus. Rev. enferm. UERJ, e7722e7722.

Zanatta, L., Dalla Cort, F. N., Mathias, N. S., \& Argenta, C. (2020). Análise das interações medicamentosas e perfil epidemiológico de indivíduos com diabetes mellitus na atenção primária. Revista de Enfermagem da UFSM, 10, 47. 